\title{
Complete retrieval of the field of ultrashort optical pulses using the angle-frequency spectrum
}

\author{
F. Bragheri, ${ }^{1,5}$ D. Faccio, ${ }^{2,5, *}$ F. Bonaretti, ${ }^{2,5}$ A. Lotti, ${ }^{2,5}$ M. Clerici, ${ }^{2,5}$ O. Jedrkiewicz, ${ }^{2,5}$ C. Liberale, ${ }^{3}$ \\ S. Henin, ${ }^{1}$ L. Tartara, ${ }^{1,5}$ V. Degiorgio, ${ }^{1,5}$ and P. Di Trapani ${ }^{2,4,5}$ \\ ${ }^{1}$ Department of Electronics, University of Pavia, Via Ferrata 1, I-27100 Pavia, Italy \\ ${ }^{2}$ CNISM \& Department of Physics and Mathematics, University of Insubria, Via Valleggio 11, I-22100 Como, Italy \\ ${ }^{3}$ Department of Experimental and Clinical Medicine, University of Magna Graecia, Viale Europa, \\ I-88100 Catanzaro, Italy \\ ${ }^{4}$ Department of Quantum Electronics, Vilnius University, Sauletekio Avenue 9, Building 3, LT-2040, \\ Vilnius, Lithuania \\ ${ }^{5}$ Virtual Institute for Nonlinear Optics, Centro di Cultura Scientifica Alessandro Volta, Villa Olmo, \\ Via Simone Cantoni 1, 22100 Como, Italy \\ *Corresponding author: daniele.faccio@uninsubria.it
}

\begin{abstract}
We propose an experimental technique that allows for a complete characterization of the amplitude and phase of optical pulses in space and time. By the combination of a spatially resolved spectral measurement in the near and far fields and a frequency-resolved optical gating measurement, the electric field of the pulse is obtained through a fast, error-reduction algorithm.
\end{abstract}

Ultrashort laser pulses are widely used in many laboratories and are routinely adopted for many applications. In past decades, various techniques were developed to measure the electric field of optical pulses as a function of time or frequency. The different approaches belong to two main categories: spectrographic and interferometric techniques. The most known examples of these approaches are frequencyresolved optical gating (FROG) [1] and spectral interferometry for direct electric-field reconstruction (SPIDER), respectively [2]. The first is based on the measurement of the temporally resolved spectrum (spectrogram). An iterative inversion algorithm is applied to the measured spectrogram in order to retrieve the electric field. The second approach consists of the measurement of the interference between a pair of spectrally sheared replicas of the input pulse. A direct inversion of the measured interferogram yields the electric field of the pulse.

These techniques have an analog in the spatial coordinate, and are often applied by assuming that temporal and spatial features of pulses are independent. This assumption is no longer valid for situations involving beam focusing [3], pulse shaping using zero-dispersion line [4] and compression [5], or nonlinear interactions (see, e.g., [6,7]) leading to space-time coupling effects. In the past few years some techniques have been proposed to characterize the amplitude and phase profile of pulses both in space and time. Among these, the most interesting are variants of FROG and SPIDER techniques with extension to the spatial dimension characterization. Indeed, in [8] a combination of FROG and digital holography is proposed to characterize the complete (3D) electric field of a train of laser pulses possessing at least one point in space where all the frequencies are present. This method is based on the use of a tunable filter or of a series of bandpass filters. A variation of this technique is proposed in [9], which has the advantage of being a single-shot measurement. The same authors recently proposed a technique that is based on crossed-beam spectral interferometry [10]. By exploiting the coherence between a reference pulse and the one under investigation, this allows for a characterization of the field both in time and space with high spectral resolution, but requires a highresolution scan along the spatial dimension.

On the other hand, the extension to the spatial dimension of the SPIDER technique has been proposed in [11-13]. Thanks to a direct inversion algorithm, the technique is capable of a fast reconstruction of the electric field as a function of one transverse spatial coordinate and time. If high-energy interfering pulses are available, this technique grants singleshot operation, but it demands different experimental setups if pulses with largely different durations have to be measured.

In this Letter we propose a new technique for the complete retrieval of the optical amplitude and phase using the $\left(k_{\perp}, \omega\right)$ spectrum (CROAK) in one spatial dimension and in time for ultrashort complex pulses of arbitrary duration. The technique is ideally developed for pulses with cylindrical symmetry but may also be applied, as in this work, to pulses that are space-time coupled only along one transverse dimension. The method is based on the measurement of the frequency-resolved near and far fields of the pulse, i.e., $(r, \omega)$ and $\left(k_{\perp}, \omega\right)$ spectra, respectively. From these spectra we obtain the spatial phases for each frequency of the pulse, which are finally linked together by a single FROG measurement. The technique exploits a sequence of two phase-retrieval algorithms, already known in the literature, which allows for a fast and accurate reconstruction of the amplitude and phase of the pulse.

The proposed technique is not single shot, since three different measurements are needed. However, this does not significantly complicate the experimen- 
tal setup, as slight modifications of the same layout are sufficient to obtain all the necessary experimental data. The experimental layout is shown in Fig. 1. The setup is very similar to that of the well-known second-harmonic FROG (SH-FROG) with the addition of the lenses $L$ that form an image of the input pulse to be characterized onto the input facet of the KDP crystal used for second-harmonic generation. The noncollinear second-harmonic signal is then imaged by the lens $\mathrm{L}_{F}$ onto the entrance slit of an imaging spectrometer and recorded with a 16 bit CCD camera (Andor Technologies). When repeated for each value of the delay $\tau$, this measurement gives the SH-FROG spectrogram $S$ as a function of the radial position $r$, i.e., $S=S(r, \lambda, \tau)$. The mirror M may then be moved in order to intercept beam 2 instead of beam 1 and allow measurement of the timeintegrated near-field spectrum $I(r, \lambda)$. Finally, by moving the lens $\mathrm{L}_{F}$ to a distance from the spectrometer equal to its focal length, the time-integrated farfield spectrum $I(\theta, \lambda)$ is measured and subsequently expressed in the function of the variables $\left(k_{\perp}, \omega\right)$. Once all data are collected, we use an error-reduction algorithm to retrieve the spatial phase for each wavelength of the pulse. The retrieval algorithm is based on the error-reduction, or Gerchberg-Saxton, algorithm [14]. It consists of four steps: (1) Fourier transform the electric field profile $E(r, \omega)$, whose amplitude is obtained by the experimental intensity while the phase is an initial guess; (2) replacement of the modulus of the resulting computed Fourier transform with the measured $\left|E\left(k_{\perp}, \omega\right)\right|$ profile; (3) inverse Fourier transform; and (4) replacement of the modulus of the resulting computed field $E(r, \omega)$ with the measured one to form a new estimate of the object. Convergence is verified by comparing the retrieved $\left(k_{\perp}, \omega\right)$ spectrum with the measured $\left(k_{\perp}, \omega\right)$ spectrum. This procedure gives us the amplitude and phase in $r$ for each frequency. The electric field may be written as

$$
E(r, \omega)=|E(r, \omega)| e^{i\left[\varphi(r, \omega)+\varphi_{0}(\omega)\right]} .
$$

We note that error reduction is applied only along the spatial dimension so that each frequency slice is independent of the other. In other words, there is a ran-

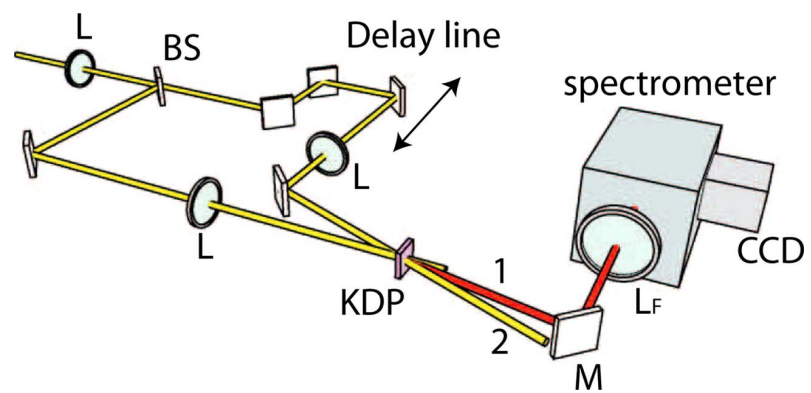

Fig. 1. (Color online) Experimental layout of the setup used in the three steps of the technique. Lenses $\mathrm{L}$ form a telescope that images the object to be measured onto the nonlinear KDP crystal. Lens $\mathrm{L}_{F}$ is moved so as to image the output facet of KDP or to give the object's far field at the entrance of the spectrometer. M, mirror. dom phase jump between each frequency slice $\varphi_{0}(\omega)$; this can be eliminated by renormalizing the phase at each $\omega$ for a fixed spatial position $r$ (e.g., $r=0$ ) to the phase obtained at the same coordinate $r$ from the FROG measurement $S(0, \lambda, \tau)$. The phase profiles at all other incidents of $r$ will therefore also be correctly aligned, and we obtain the full $(r, t)$ amplitude and phase of the pulse by performing a final temporal Fourier transform.

We verified the technique by characterizing a tilted pulse [15]. Experiments were performed with a $1.2 \mathrm{ps}, 1055 \mathrm{~nm}$ laser pulse delivered by a $2 \mathrm{~Hz}$, amplified Nd:glass laser (Twinkle, Light Conversion Ltd.). The tilted pulse was obtained by means of a grating that induces angular dispersion on the incoming radiation. In particular, the laser pulse was directed on an 1800 lines $/ \mathrm{mm}$ grating, producing a front tilt of $82.5^{\circ}$, as shown schematically in Fig. 2(a). The imaging lenses $\mathrm{L}$ were placed so as to image a plane $2 \mathrm{~cm}$ after the grating onto the KDP crystal input facet.

The measured spectrogram at $r=0$ and intensity profiles of the near- and far-field spectra are shown in Figs. 2(b)-2(d), whereas the spatiotemporal profile of the pulse reconstructed through the retrieval algorithm is shown in Fig. 3. In particular, Fig. 3(a) shows the normalized amplitude of the retrieved pulse as a function of time $t$ and space $r$, whereas Fig. 3 (b) reports the retrieved phase profile shown only in correspondence of the pulse. Optimal convergence was obtained with an initial guess function having a quadratic spatial phase curvature, and repeated computational trials showed convergence to the same spatial phase distribution or its phase-conjugated one. From the first plot we observe that the algorithm has correctly retrieved the tilted pulse with a spacetime coupling given by $r=\gamma \cdot t$. The constant parameter $\gamma$ is related to the tilt angle, which is found to be $82^{\circ}$, in extremely good agreement with the expected value. On the other hand, the second plot shows a parabolic behavior of the phase along the pulse with a maximum in correspondence of the central spatial and temporal coordinates. The curvature of the spatial phase, which may also be derived directly from
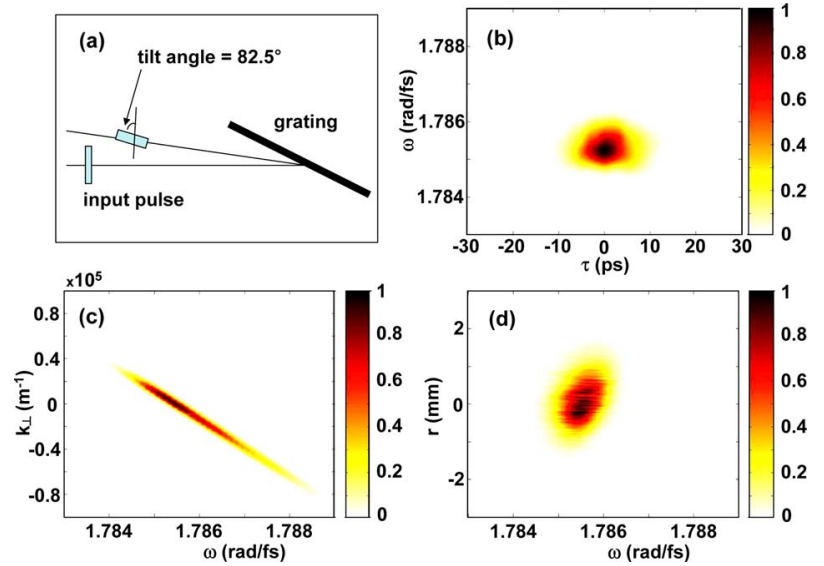

Fig. 2. (Color online) (a) Tilted pulse produced by the grating. Experimental data: (b) measured spectrogram, (c) farfield spectrum, (d) near-field spectrum. 

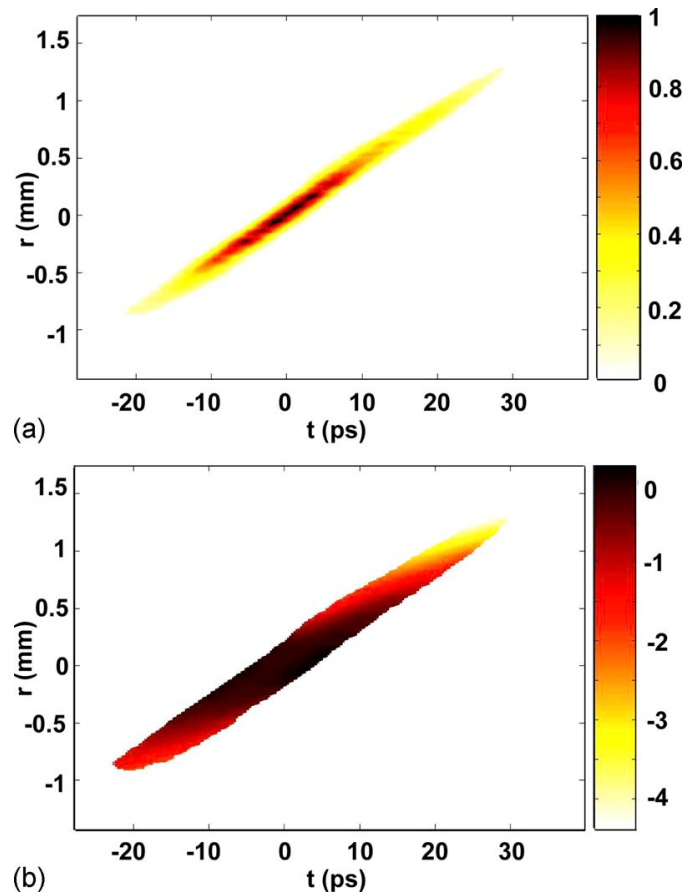

Fig. 3. (Color online) (a) Retrieved amplitude and (b) phase of the TP in function of time and space. The energy of the pulse lies on a straight line $r=\operatorname{cost} \cdot t$ and the phase shows a quadratic chirp.

the output of the error-reduction algorithm, has a radius of $56 \mathrm{~m}$ along the tilt direction and is given by the input laser beam. We note that a parabolic phase in the transverse dimension may be expected after reflection from a grating [16]. Evaluating this phase curvature analytically [16], we find that at a distance of $2 \mathrm{~cm}$ after the grating (corresponding to the plane imaged onto the nonlinear crystal) we obtain a curvature with a radius of $215 \mathrm{~m}$, which is relatively large and is not visible in our measurements.

Our data show that the proposed technique can correctly retrieve the amplitude and phase of strongly coupled space-time pulses. It is worth mentioning that simple pulses such as a collimated Gaussian beam also can be characterized by choosing an appropriate lens to perform the far-field measurement. We emphasize that although it is not single shot, the technique requires a simple setup and shares the same advantages of standard FROG, such as the possibility of characterizing even very lowintensity pulses with largely different durations without the need to modify any of the optical elements. We also note that the measurements described here use only a single FROG measurement at a given $r$, although use of the CCD gives the FROG traces at all $r$. This is acceptable only if, at this $r$, all of the pulse frequencies are present simultaneously. In any case, this limitation may be easily removed by using FROG measurements at more than one $r$ by taking care that the overlap of the obtained spectra covers the full pulse spectrum. No additional measurements are required due to the fact that the FROG traces are measured at different spatial coordinates using a CCD.

In conclusion, we propose a new technique based on the combination of FROG $(r, \lambda)$ and $(\theta, \lambda)$ spectra measurements to completely characterize in space and time the amplitude and phase of an ultrashort pulse. The novelty of the method consists mainly in linking the two spatial dimensions, i.e., near and far field, exploiting the two integrated measurements. By means of these we can reconstruct the phase profile through a fast error-reduction algorithm. Moreover, the same experimental arrangement can be used to characterize shorter pulses, which, having broader spectra, would benefit from the high resolution achievable through the use of the imaging spectrometer.

The authors gratefully acknowledge the financial support from CNISM, the INNESCO project, the RBIN04NYLH project, and the Lithuanian Science and Technology Foundation Project ConTex. P. Di Trapani acknowledges the EU Marie Curie Chair action STELLA, contract MEXC-2005-025710.

\section{References}

1. D. J. Kane and R. Trebino, IEEE J. Quantum Electron. 29, 571 (1993).

2. C. Iaconis and I. A. Walmsley, IEEE J. Quantum Electron. 35, 501 (1999).

3. Z. Bor, Opt. Lett. 14, 119 (1989).

4. M. M. Wefers and K. A. Nelson, IEEE J. Quantum Electron. 32, 161 (1996).

5. C. Fiorini, S. Sauteret, C. Rouyer, N. Blanchot, S. Seznec, and A. Migus, IEEE J. Quantum Electron. 30, 1662 (1994).

6. D. Faccio, M. A. Porras, A. Dubietis, F. Bragheri, A. Couairon, and P. Di Trapani, Phys. Rev. Lett. 96, 193901 (2006).

7. A. Couairon and A. Mysyrowicz, Phys. Rep. 441, 47 (2007).

8. P. Gabolde and R. Trebino, Opt. Express 12, 4423 (2004).

9. P. Gabolde and R. Trebino, Opt. Express 14, 11460 (2006).

10. P. Bowlan, P. Gabolde, and R. Trebino, Opt. Express 15, 10219 (2007).

11. C. Dorrer, E. M. Kosik, and I. A. Walmsley, Opt. Lett. 27, 548 (2002).

12. C. Dorrer and I. A. Walmsley, Opt. Lett. 27, 1947 (2002).

13. C. Dorrer, E. M. Kosik, and I. A. Walmsley, Appl. Phys. B 74, S209 (2002).

14. J. R. Fienup, Appl. Opt. 21, 2758 (1982).

15. S. A. Akhmanov, V. A. Vysloukh, and A. S. Chirkin, Optics of Femtosecond Laser Pulses (American Institute of Physics, 1992).

16. O. E. Martinez, Opt. Commun. 59, 229 (1986). 